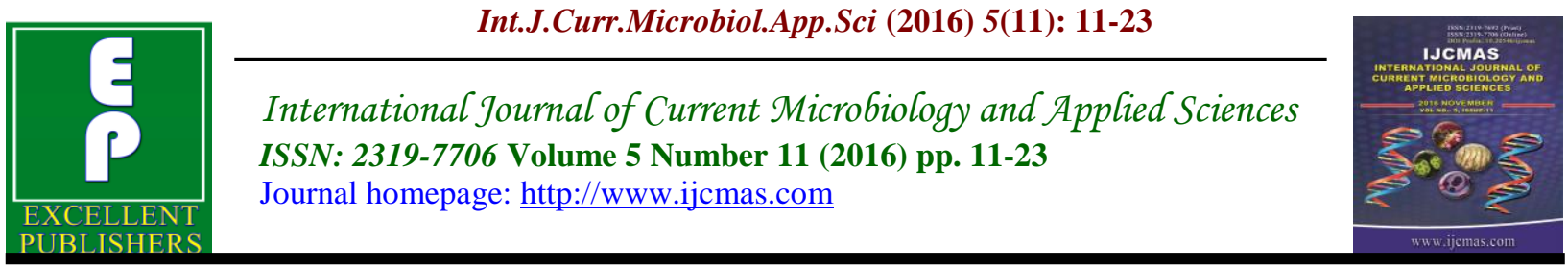

Original Research Article

http://dx.doi.org/10.20546/ijcmas.2016.511.002

\title{
Studies on Phytochemical Characteristics and Antimicrobial Activity of Pleurotus spp. Cultivated on Different Agro Wastes
}

\author{
Arvind K. Sharma ${ }^{1}$, Archana Srivastav ${ }^{2}$ and Asha Mukul Jana ${ }^{3}$ \\ ${ }^{1}$ Department of Biotechnology, College of life science, Cancer Hospital and Research Institute \\ Campus, Gwalior (Madhya Pradesh) India \\ ${ }^{2}$ Department of Microbiology, College of life science, Cancer Hospital and Research Institute \\ Campus, Gwalior (Madhya Pradesh India \\ ${ }^{3}$ Retired Scientist DRDE and Former HOD of Biotechnology and College of Life Sciences, \\ CHRI Campus, Gwalior (Madhya Pradesh) India \\ *Corresponding author
}

Keywords

Peurotus spp., agro wastes materials, phytochemical screening, and Antimicrobial activity.

\section{Article Info}

Accepted:

05 October 2016

Available Online:

10 November 2016

\section{A B S T R A C T}

In the present study Mushrooms pleurotus ostreatus and pleurotus florida were cultivated on different agro wastes namely as gram $\left(S_{1}\right)$, Pea straw $\left(S_{2}\right)$ and pearl millet cuttings $\left(S_{3}\right)$ for the screening of phytochemical characteristics and antimicrobial activity. Qualitative analysis revealed the phytochemicals alkaloids, saponins, flavonoids and tannins were present in methanolic and aqueous extracts of both Pleurotus spp. while anthraquinones and phobetanins were found absent. Antimicrobial activity was carried out against human pathogenic microorganism Escherichia coli, Stphylococcus aureus, Protius mirabilis and Candida albicans. $P$.ostreatus cultivated on substrate $S_{1}$ was recorded for highest antibacterial activity against S.aureus $(18 \mathrm{~mm})$, P.mirabilis $(13.8 \mathrm{~mm})$ and E.coli $(16.2 \mathrm{~mm})$.However methanolic extract of P.florida gave strong antifungal activity against C.albicans $(12.5 \mathrm{~mm})$ when compared to P.ostreatus .Therefore results suggested that P.ostreatus and P.florida cultivated on Substrate $S_{1}$ were found with highest antimicrobial activity in comparison to other substrates. The results supported the methanolic extracts of P.ostreatus and P. florida might indeed be potential sources of phytochemicals and antimicrobial agents.

\section{Introduction}

Mushroom are the most conspicuous structures of the fungi world. Mushroom is a fleshy, spore bearing fruiting body of fungi, typically produced above ground on soil or on its food source. Mushroom belongs to phylum Basidiomycota and some of them in the Ascomycota are known as the higher fungi (Moradali et al., 2007; Sicoli et al., 2005).
The oyster mushroom Pleurotus spp. is widely cultivated on a wide range of substrates which are composed of lignin and cellulose. Cultivation of Pleurotus spp supports a broad range of temperatures (15$30^{\circ} \mathrm{C}$ ) on different range of substrates like agro waste residues, weeds and wastes after the production of food, feed, vitamins, enzymes and a number of pharmaceuticals 
in addition to their waste degradation and detoxification properties (Gregori et al., 2007; Jonathan et al., 2012).

Mushroom is being used as a valuable food source and traditional medicine around the world since ancient times especially in China and Japan. Mushrooms are rich sources of bio active compounds as Bglucan, proteoglucan, lectin, phenolic compounds, flavonoids, polysaccharides, triterpenoids, diatery fibre, lentinan, schizophyllan, lovastatin, pleuran, steroids, glycopeptides, terpenes, saponins, xanthones, coumarins, alkaloid, purin, purimidin, kinon, fenil propanoid, kalvasin, volvotoksin, flammutoksin, porisin, AHCC, maitake D-fraction, ribonucleas, eryngeolysin. Pharmacological and nutritional aspects make the mushroom as an important tool for ailment of severe diseases like antimicrobial and antiviral infections, anticancer, antitumor, antiinflamatory, cardiovascular diseases, immunomodulating diseases (Benedict and Brady, 1972; Conchran, 1978; Karacsonyi and Kuniak, 1994; Gunde-Cimerman, 1995; Wang and Ng, 2007; Iwalokun et al., 2007)

The bioactive compounds present in Pleurotus spp. makes it a medicinally important mushroom (Gregori et al., 2007). Ahmed et al., (2009) pointed out 12 species among 40 species of Pleurotus were being cultivated in different parts of India. Only 34 species of Pleurotus are tested for their pharmaceutical importance.

Pleurotus spp. is promising as medicinal mushrooms, exhibiting hematological, antiviral, antitumor, antibiotic, antibacterial, hypocholesterolic and immunomodulation activities (Cohen et al., 2004). The oyster mushroom may also be considered as medicinal mushroom for its hypocholesterolic property, because it contains statins such as lovastatin which reduces cholesterol (Gunde-Cimerman, 1995).

In recent years, high scale usage of synthetic antibiotic leads the emergence of multi drug resistance pathogens, is now posing a threat to the world. Therefore, a search for natural plant based antimicrobial agents is in need. This development is the consequence of the limited effectiveness of synthetic products to fight against newer and drug resistant bacteria. For this purpose, the antimicrobial properties of many natural compounds from a wide variety of plant species have been assessed (Karuppusamy, 2009).Therefore this study was done to evaluate the effect of different substrate on the phytochemicals and antimicrobial activity of P.ostreatus and P.florida.

\section{Materials and Methods}

This study was carried out to evaluate the antimicrobial activity of mushroom after cultivation on different substrate.

\section{Spawn collection}

The mother spawn of Pleurotus florida and Pleurotus ostreatus were purchased from Directorate of Mushroom Research, Chambaghat, (Himachal Pradesh) India.

\section{Spawn preparation}

Spawn was prepared by using method of Bano and Shrivastava (1962) with slight modifications. Take one $\mathrm{kg}$ of wheat grain and cooked for 40 min after than washed in tap-water. Grain was drained and supplemented with $2 \mathrm{~g}$ lime and $8 \mathrm{~g}$ gypsum and mixed manually. Then grain was filled in poly propylene (PP) bags of $1 \mathrm{Kg}$ capacity and sterilized in autoclave at $121^{\circ} \mathrm{C}$ for $15 \mathrm{~min}$. After cooling, PP bag was inoculated with freshly prepared mycelium (previously prepared PDA plate) and 
incubated at $25^{\circ} \mathrm{C}$ for two weeks in an incubator.

\section{Cultivation}

Cultivation of mushroom Pleurotus spp. were carried out at College of life sciences and CHRI, Gwalior, Madhya Pradesh (India). Three different agricultural wastes were selected for cultivation as Gram straw $\left(\mathrm{S}_{1}\right)$, Pea Straw $\left(\mathrm{S}_{2}\right)$ and Pearl Millet cuttings $\left(\mathrm{S}_{3}\right)$ were collected from rural areas of Gwalior region.

These agro wastes were used as substrate material in present study. Pleorotus spp. namely as Pleurotus ostreatus, and Pleurotus florida are agricultural lignocellulose utilizing species. One $\mathrm{kg}$ of each substrate was filled in jute bags and sterilized chemically (Formaldehyde and carben) the substrate materials.

Each substrate was mixed with freshly prepared spawn and then filled in pre sterilized poly propylene (pp) bags which were incubated at $27 \pm 2{ }^{\circ} \mathrm{C}$ in the dark cultivation room for 2 to 3 weeks or until the mycelium completely colonized the substrate material. $P$. ostreatus, and $P$. florida need different incubation temperature. Humidity ( $80-85 \%$ ) of culture room was maintained by spraying water on pp bags once or twice day. Fruiting bodies were harvested after the maturation.

\section{Preparation of the mushroom extract}

Freshly harvested fruiting bodies from P.ostreatus and P.florida were shade dried and finely powdered. Twenty grams of the powder were extracted with $200 \mathrm{ml}$ of $95 \%$ solvent methanol, and aqueous separately using soxhlet apparatus. The remaining extract was filtered and evaporated by vacuum distillation; the filtrate thus obtained was used as mushroom extract (Jayakumar et al., 2009).

\section{Preliminary phytochemical characteristics}

Preliminary phytochemicals were qualitativly analysed by using methods of Trease and Evans (1994) and Harborne (1973) for alkaloids, tannins, saponins, anthrquinone, phlobatanenes flavonoids, steroids and glycosides.

\section{Antimicrobial activity}

Antimicrobial activity of the different extracts of P.ostreatus and P. florida mushroom were evaluated against microorganisms namely Escherichia coli (MTCC 1610), Staphylococcus aureus (MTCC 3160), Protius mirabilis (MTCC 425), and Candida albicans (MTCC 854) using agar well diffusion technique (Akpata and Akinrimisi, 1977). An overnight culture of each microbial isolate was mixed with nutrient broth to a turbidity of 0.5 McFarland $(108 \mathrm{cfu} / \mathrm{ml}) .100 \mu \mathrm{l}$ of each standard inoculum was then streaked on Mueller-Hinton and PDA (Potato Dextrose agar medium). Each mushroom extract and standard (streptomycin and fluconazole) was dissolve in DMSO (di methyl sulphoxide) in a concentration of $10 \mathrm{mg} / \mathrm{mL}$ and stored at $4^{\circ} \mathrm{C}$. Five Wells of $6 \mathrm{~mm}$ were made on the agar plate using a sterile cork borer and filled with $20 \mu \mathrm{L}, 40 \mu \mathrm{L}, 60 \mu \mathrm{L}, 80 \mu \mathrm{L}$ and $100 \mu \mathrm{L}$ of mushroom extracts. After incubation for 24 hours at $30^{\circ} \mathrm{C}$, a clear zone around a well was formed that considered as antibacterial activity. Diameter of the zones of inhibition was measured in millimeters. Solvent was used as a negative control.

\section{Result and Discussion}

The present study was carried out to know the phytochemical potentiality and 
antimicrobial activity of Pleurotus spp. (P.ostreatus and P.florida) mushrooms cultivated on different agro wastes namely Gram straw $\left(\mathrm{S}_{1}\right)$, Pea straw $\left(\mathrm{S}_{2}\right)$ and Pearl millet cuttings $\left(\mathrm{S}_{3}\right)$.

Findings of qualitative analysis of phytochemicals shown in table-1. Phytochemical analysis of P.ostreatus showed that the methanolic and aqueous extracts contain alkaloids, tannins, saponins, flavonoids, steroids, and glycosides as reported by Okwulehie and Ogoke (2013). Phytochemicals present in methanolic and aqueous extracts of .P florida was supported by studies of Menaga et al., (2012).

\section{Antimicrobial activity}

Antimicrobial activity of Pleurotus spp (P.ostreatus and P.florida) was carried out against pathogenic microorganisms namely E. coli, S. aureus, P. mirabilis, and C. albicans. Maximum activity was recorded against S.aureus was $18 \mathrm{~mm}$ showed by methanolic extract of P.ostreatus when cultivated on substrate $S_{1}$ and minimum was $7.2 \mathrm{~mm}$ by aqueous extract as shown in table 2 and 3 (graph 1).The obtained results showed similarity with the findings of Menaga et al., (2012) .In the present study, methanolic extracts of Pleurotus spp showed the activity against S.aureus $(7.6 \mathrm{~mm}-18.0$ $\mathrm{mm})$, E.coli (10.8 mm-16.2 $\mathrm{mm})$, P.mirabilis $(9.5 \mathrm{~mm}-13.8 \mathrm{~mm})$, C.albicans (7.1 mm-13.2 mm) as shown in table 2,4,6and 8(graph 1,2,3 and 4).On the other hand Aqueous extracts showed the antimicrobial activity against S.aureus $(7.2$ $\mathrm{mm}$ - $17.3 \mathrm{~mm}$ ), E.coli (10.5 mm-15.1 $\mathrm{mm})$,P.mirabilis $\quad(8.4 \quad \mathrm{~mm}-12.9 \quad \mathrm{~mm})$, C.albicans $(6.5 \mathrm{~mm}-12.6 \mathrm{~mm})$ as given in table 3,5,7 ,9 and Graph 1,2 and 3 .Mushroom obtained from Substrate $\mathrm{S}_{1}$ found with excellent antimicrobial activity whereas the mushrooms obtained from substrate $\mathrm{S}_{3}$ were recorded with poor antimicrobial activity because of low production of bioactive compounds. Methanolic extracts of P.ostreatus from substrate $S_{1}$ gave best results against E.coli $(16.2 \mathrm{~mm})$ S.aureus $(18 \mathrm{~mm})$ and P.mirabilis $(13.8 \mathrm{~mm})$.However methanolic extract of P.florida was also recorded with high antifungal activity against C.candida $(13.2 \mathrm{~mm})$. The results of Akyuz et al., (2009) on antimicrobial activity of methanolic extract of Pleurotus spp. against B. megaterium, E. coli, K. pneumonia, S. aureus, C. albicans, C. glabrata Epidermophyton spp. Trichophyton spp. explained that petroleum ether and acetone extracts of $P$. ostreatus were found effective against Staphylococcus spp. (7.0-7.6 mm), Bacillus spp. (7.1-7.8 mm), S. thyphi (7.0$7.5 \mathrm{~mm}), \quad$ E. coli $(7.0-8.2 \mathrm{~mm}), \quad K$. pneumoniae (7.0-7.1 $\mathrm{mm}$ ) and Candida spp. (8.0-8.3 mm). P.ostreatus showed high activity against C.glabrata was $15.5 \mathrm{~mm}$. Jagadish et al. (2008) reported the ethanol extract of $P$. florida and $P$. aureovillosus did not exhibit antimicrobial effect against $K$. pneumoniae, $P$. vulgaris, $P$. aeruginosa and C. albicans, but showed activity against $S$. aureus $(16.0 \mathrm{~mm}$ and $20.0 \mathrm{~mm})$, S. mutans (14.0 and $17.0 \mathrm{~mm})$, M. luteus (16.0 and $19.0 \mathrm{~mm})$, B. subtilis $(9.0$ and $14.0 \mathrm{~mm})$ and E. coli (12.0 and $14.0 \mathrm{~mm})$, respectively.

Iwalokun (2007) also reported the similar results. Mondal et al., (2013) found the inhibition zone ranged from $3.5 \mathrm{~mm}-17 \mathrm{~mm}$ was formed by extract of P.ostreatus during an antimicrobial study. Its methanolic extracts gave best results against E.coli (15.2 $\mathrm{mm})$ and S.aureus $(16.6 \mathrm{~mm})$ was very close to present study. Surekha et al., (2011) reported the antimicrobial activity of P.ostreatus against pathogenic bacteria E.coli $(15 \mathrm{~mm})$, S.aureus $(24 \mathrm{~mm})$ and P.vulgaris (18mm). 
Table.1 Qualitative analysis of phytochemicals

\begin{tabular}{|c|c|c|c|c|c|c|c|c|c|c|}
\hline \multirow{2}{*}{$\begin{array}{c}\text { Mushroom } \\
\text { Species }\end{array}$} & \multirow{2}{*}{ Substrate } & \multirow{2}{*}{ Extract } & \multicolumn{8}{|c|}{ Phytochemicals } \\
\hline & & & Alkaloids & Anthraquinones & Saponins & Flavonoids & steroids & $\begin{array}{c}\text { phlobatannin } \\
\text { S }\end{array}$ & tannins & glycosides \\
\hline \multirow{6}{*}{ P.ostreatus } & \multirow[t]{2}{*}{$\mathrm{S}_{1}$} & Methanolic & + & - & + & + & + & - & + & + \\
\hline & & Aqueous & + & - & + & + & - & - & + & - \\
\hline & \multirow[t]{2}{*}{$\mathrm{S}_{2}$} & Methanolic & + & - & + & + & + & - & + & + \\
\hline & & Aqueous & + & - & + & + & - & - & + & - \\
\hline & \multirow[t]{2}{*}{$\mathrm{S}_{3}$} & Methanolic & + & - & + & + & + & - & + & + \\
\hline & & Aqueous & + & - & + & + & - & - & + & - \\
\hline \multirow{6}{*}{ P.florida } & \multirow[t]{2}{*}{$\mathrm{S}_{1}$} & Methanolic & + & - & + & + & + & - & + & + \\
\hline & & Aqueous & - & - & + & + & - & - & + & - \\
\hline & \multirow[t]{2}{*}{$\mathrm{S}_{2}$} & Methanolic & + & - & + & + & + & - & + & + \\
\hline & & Aqueous & - & - & + & + & - & - & + & - \\
\hline & \multirow[t]{2}{*}{$\mathrm{S}_{3}$} & Methanolic & + & - & + & + & + & - & + & + \\
\hline & & Aqueous & - & - & + & + & - & - & + & - \\
\hline
\end{tabular}

Table.2 Antibacterial activity of methanolic extract of Pleurotus spp. cultivated on different substrates

\begin{tabular}{|c|c|c|c|c|c|c|c|c|}
\hline \multirow{3}{*}{$\begin{array}{l}\text { Bacteria } \\
\text { used }\end{array}$} & \multirow{3}{*}{$\begin{array}{c}\text { Volume of extract } \\
\text { per well }(\boldsymbol{\mu L})\end{array}$} & \multicolumn{7}{|c|}{ zone of inhibition in ( mm) } \\
\hline & & \multicolumn{3}{|c|}{ P.ostreatus } & \multicolumn{3}{|c|}{ P.florida } & Control \\
\hline & & Substrate $S_{1}$ & Substrate $S_{2}$ & Substrate $\mathbf{S}_{3}$ & Substrate $S_{1}$ & Substrate $S_{2}$ & Substrate $S_{3}$ & Streptomycin \\
\hline \multirow{5}{*}{ S. aureus } & 20 & 13.9 & 13.4 & 7.6 & 13.5 & 13.3 & 9.5 & 24.5 \\
\hline & 40 & 14.8 & 13.7 & 8.4 & 14.6 & 14.2 & 10.2 & 25.8 \\
\hline & 60 & 16 & 15.4 & 9.1 & 15.4 & 15.1 & $\mathbf{1 0 . 8}$ & 27 \\
\hline & 80 & $\mathbf{1 7 . 1}$ & 16.1 & 9.5 & 16.3 & 15.8 & $\mathbf{1 1 . 8}$ & 28.5 \\
\hline & 100 & 18 & 17.4 & $\mathbf{1 0 . 5}$ & $\mathbf{1 7 . 1}$ & 16.3 & 13.5 & 30 \\
\hline
\end{tabular}

Where, Substrate $S_{1}=$ Gram straw, $S_{2}=$ Peas straw, $S_{3}=$ Pearl millet cuttings; Concentration of extract $(10 \mathrm{mg} / \mathrm{mL})$ 
Table.3 Antibacterial activity of aqueous extract of Pleurotus spp. cultivated on different substrates

\begin{tabular}{|c|c|c|c|c|c|c|c|c|}
\hline \multirow{3}{*}{$\begin{array}{l}\text { Bacteria } \\
\text { used }\end{array}$} & \multirow{3}{*}{$\begin{array}{c}\text { Volume of extract } \\
\text { per well }(\mu \mathrm{L})\end{array}$} & \multicolumn{7}{|c|}{ zone of inhibition in ( $\mathrm{mm}$ ) } \\
\hline & & \multicolumn{3}{|c|}{ P.ostreatus } & \multicolumn{3}{|c|}{ P.florida } & Control \\
\hline & & Substrate $S_{1}$ & Substrate $\mathbf{S}_{2}$ & Substrate $S_{3}$ & Substrate $S_{1}$ & Substrate $\mathbf{S}_{2}$ & Substrate $\mathbf{S}_{\mathbf{3}}$ & Streptomycin \\
\hline \multirow{5}{*}{ S.aureus } & 20 & 13.5 & 13.1 & 7.2 & 12.9 & 12.7 & 9.1 & 18 \\
\hline & 40 & 14.2 & 13.9 & 7.9 & 14 & 13.5 & 9.9 & 20.5 \\
\hline & 60 & 14.9 & 14.8 & 8.5 & 14.8 & 14.3 & 10.5 & 21 \\
\hline & 80 & 16.5 & 16 & 9.1 & 15.2 & 14.9 & 11 & 22.8 \\
\hline & 100 & 17.3 & 16.9 & 10.2 & 16 & 15.8 & 12.7 & 25 \\
\hline
\end{tabular}

Where, Substrate $S_{1}=$ Gram straw, $S_{2}=$ Pea straw, $S_{3}=$ Pearl millet cuttings; Concentration of extract $(10 \mathrm{mg} / \mathrm{mL})$

Table.4 Antibacterial activity of methanolic extract of Pleurotus spp. cultivated on different substrates

\begin{tabular}{|c|c|c|c|c|c|c|c|c|}
\hline \multirow{3}{*}{$\begin{array}{l}\text { Bacteria } \\
\text { used }\end{array}$} & \multirow{3}{*}{$\begin{array}{c}\text { Volume of extract } \\
\text { per well }(\mu L)\end{array}$} & \multicolumn{7}{|c|}{ zone of inhibition in ( $\mathrm{mm})$} \\
\hline & & \multicolumn{3}{|c|}{ P.ostreatus } & \multicolumn{3}{|c|}{ P.florida } & Control \\
\hline & & Substrate $S_{1}$ & Substrate $S_{2}$ & Substrate $S_{3}$ & Substrate $S_{1}$ & Substrate $S_{2}$ & Substrate $\mathbf{S}_{3}$ & Streptomycin \\
\hline \multirow{5}{*}{ P.mirabilis } & 20 & 11 & - & - & 10.8 & - & - & 18 \\
\hline & 40 & 11.8 & 11.5 & - & 11.2 & 10.7 & - & 20 \\
\hline & 60 & 12.2 & 12 & 9.9 & 11.9 & $\mathbf{1 1 . 5}$ & 9.5 & 21.5 \\
\hline & 80 & 12.9 & 12.8 & 10.6 & 12.3 & 12.1 & 10.3 & 22.8 \\
\hline & 100 & 13.8 & 13.4 & $\mathbf{1 1 . 1}$ & 13.1 & 12.9 & 11.9 & 23.1 \\
\hline
\end{tabular}

Where, Substrate $S_{1}=$ Gram straw, $S_{2}=$ Pea straw, $S_{3}=$ Pearl millet cuttings; Concentration of extract $(10 \mathrm{mg} / \mathrm{mL})$

Table.5 Antibacterial activity of aqueous extract of Pleurotus spp. cultivated on different substrates

\begin{tabular}{|c|c|c|c|c|c|c|c|c|}
\hline \multirow{3}{*}{$\begin{array}{l}\text { Bacteria } \\
\text { used }\end{array}$} & \multirow{3}{*}{$\begin{array}{c}\text { Volume of extract } \\
\text { per well }(\mu L)\end{array}$} & \multicolumn{7}{|c|}{ zone of inhibition in ( $\mathrm{mm})$} \\
\hline & & \multicolumn{3}{|c|}{ P.ostreatus } & \multicolumn{3}{|c|}{ P.florida } & \multirow{2}{*}{$\begin{array}{c}\text { Control } \\
\text { Streptomycin }\end{array}$} \\
\hline & & Substrate $S_{1}$ & Substrate $\mathbf{S}_{2}$ & Substrate $S_{3}$ & Substrate $S_{1}$ & Substrate $S_{2}$ & Substrate $S_{3}$ & \\
\hline \multirow{5}{*}{ P.mirabilis } & 20 & 9.9 & 9.5 & - & 9.5 & 9.2 & - & 11 \\
\hline & 40 & 10.5 & 10.4 & 8.4 & 10 & 10.9 & - & 12.9 \\
\hline & 60 & 11.4 & 11.5 & 9.2 & 11.4 & 11.1 & 9.3 & 13.1 \\
\hline & 80 & 12.1 & 11.9 & 10.1 & 11.9 & 11.8 & 10.2 & 16.9 \\
\hline & 100 & 12.9 & 12.2 & 10.9 & 12.3 & 12.1 & 11.1 & 18.5 \\
\hline
\end{tabular}

Where, Substrate $S_{1}=$ Gram straw, $S_{2}=$ Pea straw, $S_{3}=$ Pearl millet cuttings; Concentration of extract $(10 \mathrm{mg} / \mathrm{mL})$ 
Table.6 Antibacterial activity of methanolic extract of Pleurotus spp. cultivated on different substrates

\begin{tabular}{|c|c|c|c|c|c|c|c|c|}
\hline \multirow{3}{*}{$\begin{array}{c}\text { Bacteria } \\
\text { used }\end{array}$} & \multirow{3}{*}{$\begin{array}{c}\text { Volume of extract } \\
\text { per well }(\mu \mathrm{L})\end{array}$} & \multicolumn{7}{|c|}{ zone of inhibition in ( $\mathrm{mm})$} \\
\hline & & \multicolumn{3}{|c|}{ P.ostreatus } & \multicolumn{3}{|c|}{ P.florida } & \multirow{2}{*}{$\begin{array}{c}\text { Control } \\
\text { Streptomycin }\end{array}$} \\
\hline & & Substrate $S_{1}$ & Substrate $S_{2}$ & Substrate $S_{3}$ & Substrate $S_{1}$ & Substrate $S_{2}$ & Substrate $S_{3}$ & \\
\hline \multirow{5}{*}{ E.coli } & 20 & 11.5 & 11.1 & 10.3 & 11.1 & 10.8 & - & 16 \\
\hline & 40 & 12.9 & 11.9 & 11.1 & 11.8 & $\mathbf{1 1 . 5}$ & 11 & 20 \\
\hline & 60 & 14.8 & 13.5 & 12.9 & $\mathbf{1 3 . 5}$ & 12.9 & 12.1 & 22 \\
\hline & 80 & 15.6 & 14.2 & 13.8 & 14.1 & 13.8 & 12.9 & 23 \\
\hline & 100 & 16.2 & 15 & 14.5 & 15.9 & 14.7 & 14.7 & 25 \\
\hline
\end{tabular}

Where, Substrate $S_{1}=$ Gram straw, $S_{2}=$ Pea straw, $S_{3}=$ Pearl millet cuttings; Concentration of extract $(10 \mathrm{mg} / \mathrm{mL})$

Table.7 Antibacterial activity of aqueous extract of Pleurotus spp. cultivated on different substrates

\begin{tabular}{|c|c|c|c|c|c|c|c|c|}
\hline \multirow{3}{*}{$\begin{array}{l}\text { Bacteria } \\
\text { used }\end{array}$} & \multirow{3}{*}{$\begin{array}{c}\text { Volume of extract } \\
\text { per well }(\mu \mathrm{L})\end{array}$} & \multicolumn{7}{|c|}{ zone of inhibition in( $\mathrm{mm}$ ) } \\
\hline & & \multicolumn{3}{|c|}{ P.ostreatus } & \multicolumn{3}{|c|}{ P.florida } & Control \\
\hline & & Substrate $S_{1}$ & Substrate $\mathbf{S}_{2}$ & Substrate $\mathrm{S}_{3}$ & Substrate $S_{1}$ & Substrate $\mathbf{S}_{2}$ & Substrate $\mathbf{S}_{3}$ & Streptomycin \\
\hline \multirow{5}{*}{ E.coli } & 20 & 10.9 & 10.8 & - & 11 & 10.5 & - & 15 \\
\hline & 40 & 12 & 11.7 & - & 11.9 & 11.7 & - & 17 \\
\hline & 60 & $\mathbf{1 3 . 5}$ & 13.2 & $\mathbf{1 0 . 5}$ & $\mathbf{1 2 . 5}$ & 12.2 & 10.9 & 20 \\
\hline & 80 & 14.6 & 14.1 & 11.2 & 13.4 & 13.1 & 11.5 & 21 \\
\hline & 100 & 15.1 & 14.9 & 11.9 & 14.2 & 13.9 & 12.1 & 23 \\
\hline
\end{tabular}

Where, Substrate $S_{1}=$ Gram straw, $S_{2}=$ Pea straw, $S_{3}=$ Pearl millet cuttings; Concentration of extract $(10 \mathrm{mg} / \mathrm{mL})$

Table.8 Antifungal activity of methanolic extract of Pleurotus spp. cultivated on different substrates

\begin{tabular}{|c|c|c|c|c|c|c|c|c|}
\hline \multirow{3}{*}{ Fungi used } & \multirow{3}{*}{$\begin{array}{c}\text { Volume of extract } \\
\text { per well }(\mu \mathrm{L})\end{array}$} & \multicolumn{7}{|c|}{ zone of inhibition in ( $\mathrm{mm})$} \\
\hline & & \multicolumn{3}{|c|}{ P.ostreatus } & \multicolumn{3}{|c|}{ P.florida } & Control \\
\hline & & Substrate $S_{1}$ & Substrate $S_{2}$ & Substrate $S_{3}$ & Substrate $S_{1}$ & Substrate $\mathbf{S}_{2}$ & Substrate $\mathbf{S}_{3}$ & Fluconazole \\
\hline \multirow{5}{*}{ C.albicans } & 20 & 7.5 & 7.1 & 7.3 & 9.6 & 9.1 & 8.5 & 15.1 \\
\hline & 40 & 9.2 & 7.9 & 8 & 9.9 & 9.7 & 9.3 & 15.9 \\
\hline & 60 & 10.1 & 8.8 & 8.9 & 11.2 & 10.6 & 10.9 & 16.8 \\
\hline & 80 & 11 & 9.5 & 10.1 & 12.5 & $\mathbf{1 1 . 5}$ & 11.3 & 17.2 \\
\hline & 100 & 12.5 & 10.6 & 11.2 & 13.2 & 12.9 & 11.8 & 18 \\
\hline
\end{tabular}

Where, Substrate $S_{1}=$ Gram straw, $S_{2}=$ Pea straw, $S_{3}=$ Pearl millet cuttings; Concentration of extract $(10 \mathrm{mg} / \mathrm{mL})$ 
Table.9 Antifungal activity of aqueous extract of Pleurotus spp. cultivated on different substrates

\begin{tabular}{|c|c|c|c|c|c|c|c|c|}
\hline \multirow{3}{*}{ Fungi used } & \multirow{3}{*}{$\begin{array}{c}\text { Volume of extract } \\
\text { per well }(\boldsymbol{\mu L})\end{array}$} & \multicolumn{7}{|c|}{ zone of inhibition in ( $\mathrm{mm})$} \\
\hline & & \multicolumn{3}{|c|}{ P.ostreatus } & \multicolumn{3}{|c|}{ P.florida } & Control \\
\hline & & Substrate $S_{1}$ & Substrate $S_{2}$ & Substrate $S_{3}$ & Substrate $S_{1}$ & Substrate $S_{2}$ & Substrate $S_{3}$ & Fluconazole \\
\hline \multirow{5}{*}{ C.albicans } & 20 & 7.1 & 6.5 & 6.9 & 7.8 & 7.0 & 7.2 & 15.1 \\
\hline & 40 & 8.9 & 7.2 & 7.8 & 9.4 & 7.9 & 8.5 & 15.9 \\
\hline & 60 & 9.5 & 7.8 & 8.5 & 10.9 & 8.5 & 9 & 16.8 \\
\hline & 80 & 10.4 & 8.7 & 9.6 & $\mathbf{1 1 . 5}$ & 9.7 & 10.2 & 17.2 \\
\hline & 100 & 11.2 & 10 & 10.5 & 12.6 & 10.4 & 10.8 & 18 \\
\hline
\end{tabular}

Where, Substrate $S_{1}=$ Gram straw, $S_{2}=$ Pea straw, $S_{3}=$ Pearl millet cuttings; Concentration of extract $(10 \mathrm{mg} / \mathrm{mL})$

Graph.1 Antibacterial activities of Pleurotus spp cultivated on different substrates

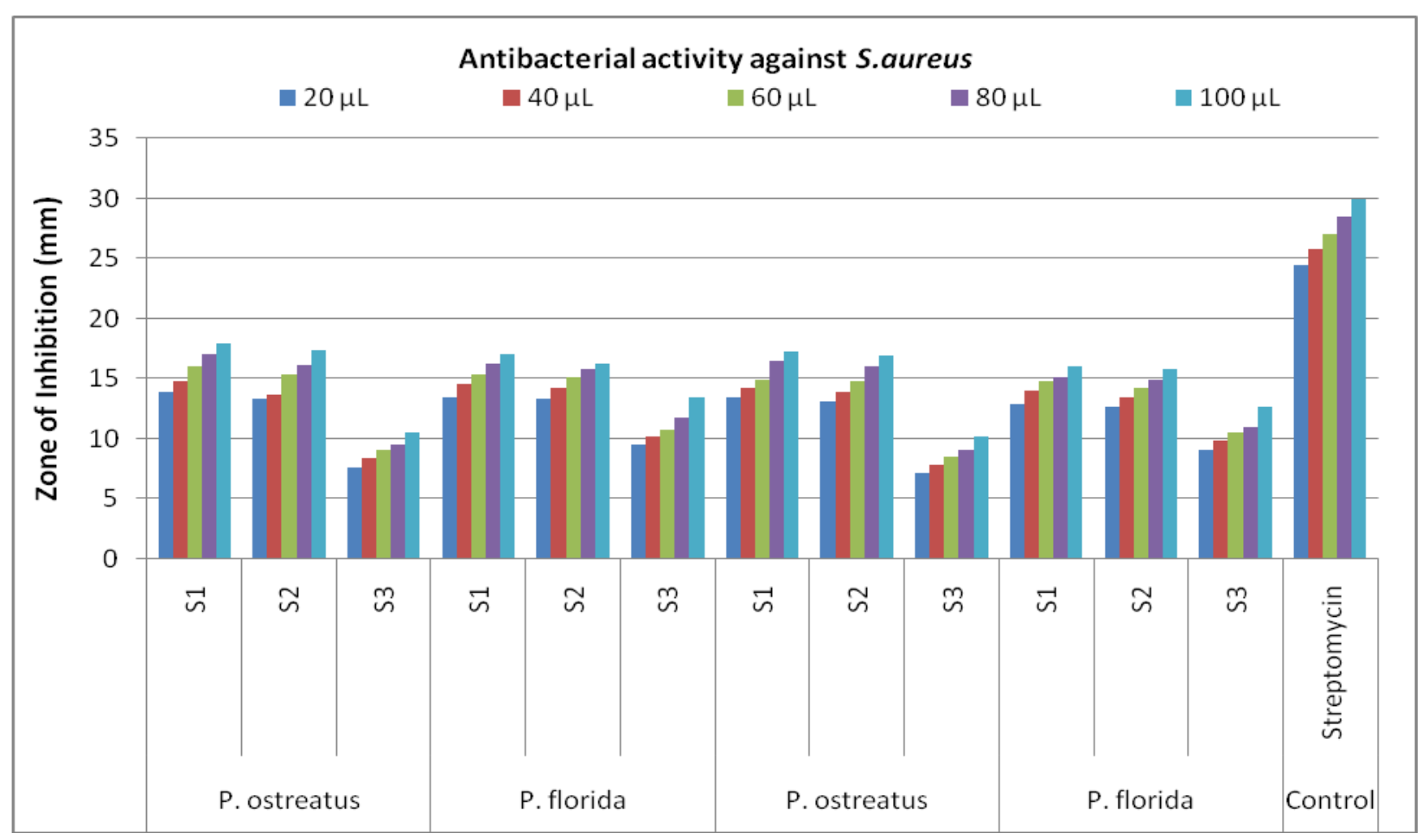


Graph.2 Antibacterial activities of Pleurotus spp cultivated on different substrates

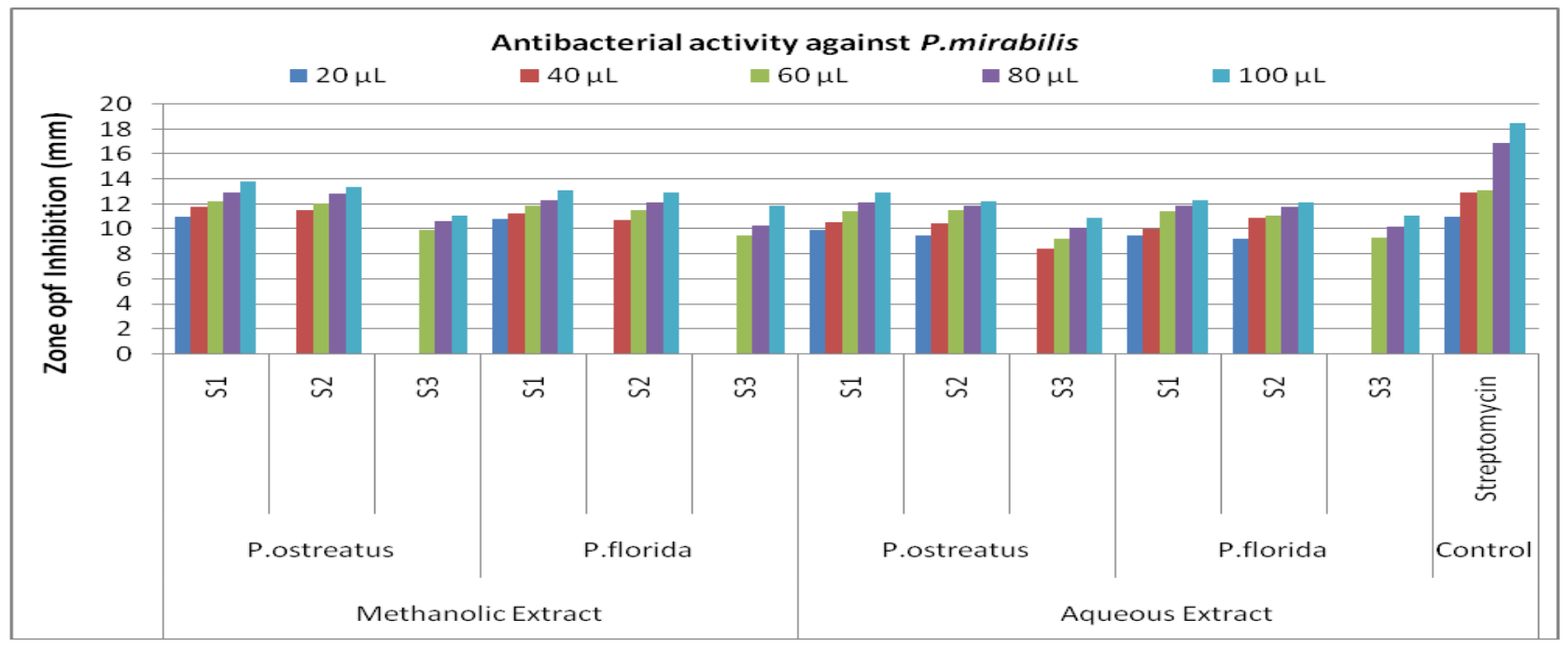

Graph.3 Antibacterial activities of Pleurotus spp cultivated on different substrates

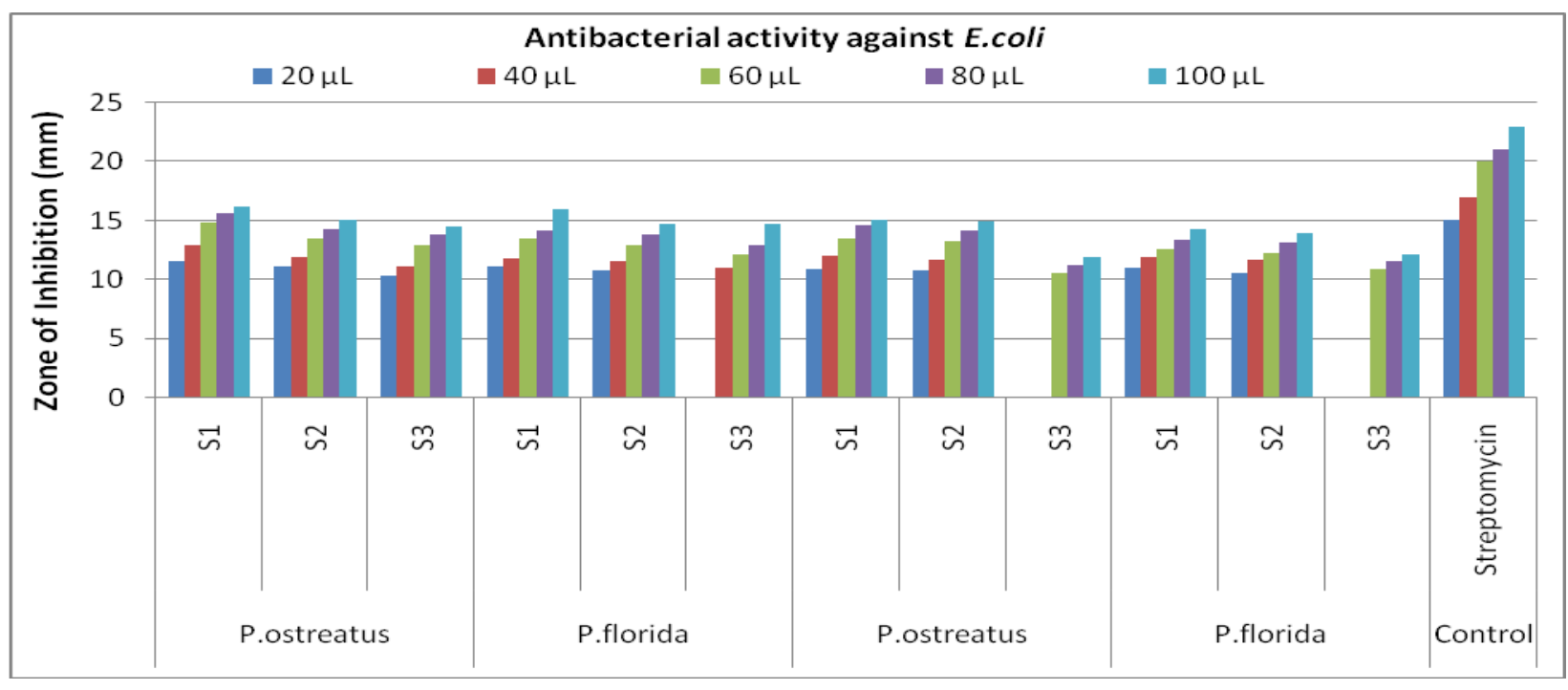


Int.J.Curr.Microbiol.App.Sci (2016) 5(11): 11-23

Graph.4 Antibacterial activities of Pleurotus spp. cultivated on different substrates

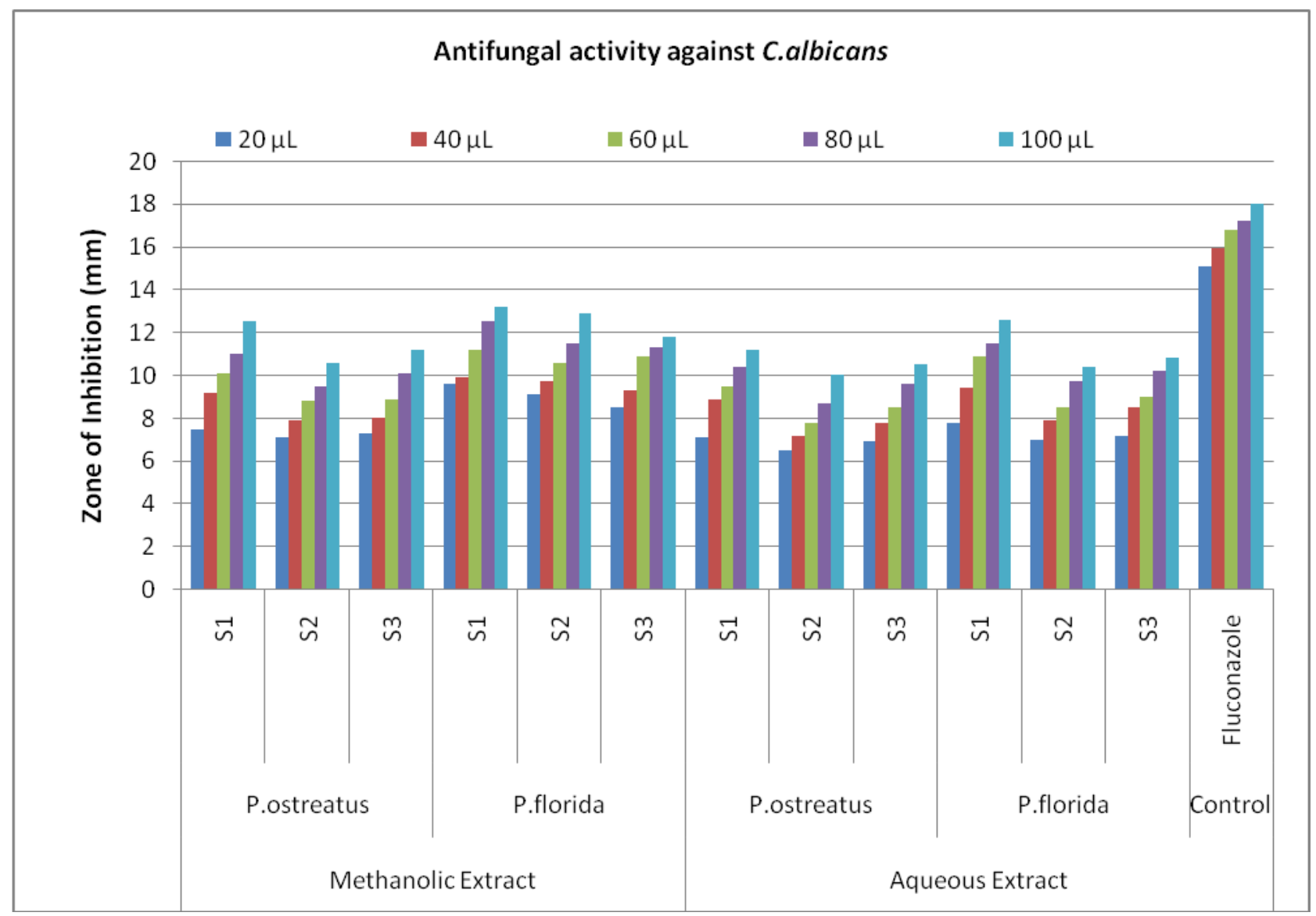


Thillaimaharani et al., (2013) reported antibacterial activity of different extracts of $P$. florida were tested against 8 human bacterial pathogens E. coli, S. typhi, K. pneumoniae, $V$. parahaemolyticus, $K$. oxytoca, P. murabilus, $V$. cholarae and Streptococcus spp. antibacterial activity of ethanol extract of $P$. florida was found Maximum (23 mm) against Streptococcus spp . and minimum $4 \mathrm{~mm}$ against $V$. parahaemolyticus. Antimicrobial activity against E. coli was found $11 \mathrm{~mm}$. Akyuz and Kirbag (2009) reported the same results for ethanol extract of $P$. eryngii showed maximum antifungal activity against $C$. albicans $(7.7 \mathrm{~mm}), C$. albicans $(7.7 \mathrm{~mm}), C$. glabrata (7.7-9.3 mm), Epidermopyton $\mathrm{sp}$. (7.7-8 $\mathrm{mm})$ and Trichophyton spp. (7.7-8.7 $\mathrm{mm})$.

Previous study of Menaga et al., (2012) on antimicrobial activity of ethanolic extract of P.florida exhibited highest activity against Pseudomonas spp. and Campylobacter spp. whereas methanol extract showed higher activity against E.coli, Salmonella typhi, Staphylococcus aureus, Camphylobacter sp., and Vibrio sp. aqueous extract also revealed high zone formation against Vibrio $s p$ was $24 \pm 1.5 \mathrm{~mm}$. Ethyl acetate and hexane extract showed highest antibacterial potency against Staphylococcus aureus and Pseudomonas spp., respectively. Menaga et al., (2012) concluded that methanol extract showed activity against E.coli $(21 \pm 0.9$ $\mathrm{mm})$, Salmonella typhi $(20 \pm 0.5 \mathrm{~mm})$, Staphylococcus aureus $(20 \pm 0.4 \mathrm{~mm})$, Camphylobacter spp. (19 $\pm 0.8 \mathrm{~mm})$, Bacillus spp. $(14 \pm 0.5 \mathrm{~mm})$, Pseudomonas spp. (8 $\pm 0.5 \mathrm{~mm})$, Klebsiella spp. (12 \pm 0.6 $\mathrm{mm})$ and Vibrio spp. $(20 \pm 0.9 \mathrm{~mm})$. In a previous study, Jonathan (2007) reported that the sporophore methanolic extract of Pleurotus florida showed activity in E.coli (13 mm), Klebsiella spp. (20 mm) and no activity against Bacillus spp., Pseudomonas and Proteus spp. 30. Menaga et al., (2012) reported the zone formation in Pseudomonas spp., $(20 \pm 0.6 \mathrm{~mm})$, Salmonella spp., $(20 \pm$ $0.5 \mathrm{~mm})$ and Klebsiella pneumonae (13 \pm $0.8 \mathrm{~mm}$ ) whereas ethanolic extract of mycelium showed zone formation in Staphylococcus aureus (16 $\mathrm{mm})$, Streptococcus mutans (14 mm), Escherichia coli $(12 \mathrm{~mm})$, Micrococcus luteus (16 mm), Bacillus subtilis $(9 \mathrm{~mm})$ and no zone formation against Pseudomonas aeruginosa, Salmonella abony, Klebsiella pneumoniae, Proteus vulgaris, Candida albicans.

In conclusion, in present study mushroom obtained from substrate (Gram straw, Pea straw and Pearl millet cuttings) showed strong antimicrobial activity against Pathogenic microorganism. Moreover, mushroom species can be used as easily available source of natural antimicrobial agent for gram negative, gram positive bacteria and pathogenic fungi. Results showed that methanol is a good solvent for extraction. Used substrate can be used for commercial cultivation of mushroom.

\section{References}

Ahmed, S.A., Kadam, J.A., Patil, S.S., and Baig, M.M.V. 2009. Biological efficiency and nutritional contents of Pleurotus florida (Mont.) Singer cultivated on different agro-wastes. Nat. Sci., 7: 44-48.

Akyuz, M., Kirbag, S. 2009. Antimicrobial activity of Pleurotus eryngii var. ferulae grown on various agro-wastes. Eur. Asian J. Biosci., 3: 58-63.

Bano, Z., and Srivastava, H.C. 1962. Studies in the cultivation of Pleurotus sp. on paddy straw. Food Sci., 12: 363-368.

Benedict, R.G., Brady, L.R. 1972. "Antimicrobial activity of mushroom metabolites", J. Pharmaceut Sci., 61(11): 1820-1822. 
Cohen, R., Persky, L., and Hadar, Y. 2004. Biotechnological applications and potential of wood-degrading mushrooms of the genus Pleurotus. Appl. Microbial. Biotechnol., 58, 582594.

Cohen, R., Persky, L., and Hadar, Y.2002. "Biotechnological applications and potential of wood-degrading mushrooms of the genus Pleurotus", Appl. Microbiol. Biotechnol., 58: 582594.

Collins, C.H., and Lyne, P.M. 1987. Microbiological Methods. Butter Worths and Co (Publishers) Ltd., London.

Collins, R.A., Ng, T.B. 1997. Polysaccharopeptide from Coriolus versicolor has potential for use against human immunodeficiency virus type I infection', Life Sci., 60(25): 383-387.

Conchran, K.W. 1978. "Medicinal effects, In: The Biology and Cultivation of Edible Mushroom", S.T., Chang, W.A., Hayes (eds.), Academic Press, New York, 160-187.

Gregori, A., Svagel, M.,and Pohleven, J. 2007. Cultivation techniques and medicinal properties of Pleurotus spp. Food Technol. Biotechnol., 45: 238249.

Gunde Cimerman, N., and Cimerman, A. 1995. Pleurotus fruiting bodies contain the inhibitor of 3-hydroxy-3methylglutaryl-coenzyme A reductaselovastatin. Exp. Mycol., 19(1): 1-6.

Harborne, J.B. 1973. Phytochemical Methods. Chapman and Hall Ltd, London. 11-113.

Iwalokun, B.A., Usen, U.A., Otunba, A.A., Olukoya, D.K.2007. "Comparative phytochemical evaluation, antimicrobial and antioxidant properties of Pleurotus ostreatus", Afr. J. Biotechnol., 6 (15): 1732-1739.
Jagadish, L.K., Shenbhagaraman, R., Venkatakrishnan, V., Kaviyarasan, V.2008."'Studies on the phytochemical, antioxidant and antimicrobial properties of three indigenous Pleurotus Species", J. Mol. Biol. Biotechnol., 1: 20-29.

Jayakumar, T., Thomas, P.A., and Geraldine, P. 2009. In-vitro antioxidant activities of an ethanolic extract of the oyster mushroom, Pleurotus ostreatus. Innovative Food Sci. Emerging Technol., 10, 228-234.

Jonathan, G. 2007. Antagonistic effect of extracts of some Nigerian higher fungi against selected pathogenic microorganisms. American-Eurasian J. Agric. \& Environ. Sci., 4: 364-368.

Karacsonyi, S., Kuniak, L. 1994. 'Polysaccharides of Pleurotus ostreatus: Isolation and structure ofpleuran, an alkali-insoluble Beta-D Glucan", Carbohyd. Polymer, 24: 107-111.

Karuppusamy, S. 2009. A review on trends in production of secondary metabolites from higher plants by in vitro tissue, organ and cell cultures. J. Med. Plants Res., 3: 1222-1239.

Menaga, D., Mahalingam, P.U., Rajakumar, S., and Ayyasamy, P.M. 2012. Evaluation of phytochemical characteristics and antimicrobial activity of Pleurotus florida mushroom, Asian J. Pharm. Clin. Res., 5(4):102-106.

Mondal, T., Rupa, S., and Dutta, S. 2013. Studies on antioxidant and antimicrobial properties of some common mushrooms. JTBSRR, 2(1): 60-67.

Moradali, M.F., Mostafavi, H., Ghods, S., and Hedjaroude, G.A. 2007. Immunomodulating and anticancer agents in the realm of macromycetes 
fungi (macrofungi). Int. Immunopharmacol., 7: 701-724.

Okwulehie, I.C., and Ogoke J.A. 2013. Bioactive, nutritional and heavy metal constituents of some edible mushrooms found in Abia State of Nigeria. Int. J. Appl. Microbiol. Biotechnol. Res., 1(2): 7-15.

Sicoli, G., Rana, G.L., Marino, R., Sisto, D., Lerario, P., and Luisi, N.2005. Forest Fungi as Bioindicators of a Healthful Environment and as Producers of Bioactive Metabolites Useful For Therapeutic Purposes. 1st European Cost E39 Working Group 2 Workshop: "Forest Products, Forest Environment and Human Health: Tradition, Reality, and Perspectives" Christos Gallis (editor) Firenze, Italy 20th - 22nd.
Surekha, Ch., Kaladhar, D.S.V.G.K., Raju, Srikakarlapudi, J.R., and Haseena. 2011. Evaluation of antioxidant and antimicrobial potentiality of some edible mushrooms, Int. J. Adv. Biotechnol. Res., 2(1): 130-134.

Thillaimaharani, K.A., Sharmila, K., Thangaraju, P., Karthick, M., and Kalaiselvam, M. 2013. Studies on antimicrobial and antioxidant properties of oyster mushroom Pleurotus florida. Int. J. Pharma. Sci. Res., 4(4): 1540-1545.

Trease, G.E., and Evans, W.C. 1994. Pharmacognosy Xii Ed London, Bailere London.

Wang, H., Ng, T.B.2007. ' 'Eryngin, a novel antifungal peptide from fruiting bodies of the edible mushroom Pleurotus eryngii', Peptides, 25: 1-5.

\section{How to cite this article:}

Arvind K. Sharma, Archana Srivastav and Asha Mukul Jana. 2016. Studies on Phytochemical Characteristics and Antimicrobial Activity of Pleurotus spp. Cultivated on Different Agro Wastes. Int.J.Curr.Microbiol.App.Sci. 5(11): 11-23. doi: http://dx.doi.org/10.20546/ijcmas.2016.511.002 